Oliveira MDM, Silva RCM \& Souza DL (2020). Multi objective optimization in the level controller project in a pilot plant. Research, Society and Development, 9(7): 1-22, e743974794.

\title{
Otimização multiobjetivo no projeto de controlador de nível em planta piloto
}

Multi objective optimization in the level controller project in a pilot plant

\section{Optimización multiobjetivo en el proyecto de controlador de nivel en una planta piloto}

Recebido: 21/05/2020 | Revisado: 23/05/2020 | Aceito: 27/05/2020 | Publicado: 08/06/2020

Matheus Divino Moraes Oliveira

ORCID: https://orcid.org/0000-0003-3301-230X

Universidade Federal do Triângulo Mineiro, Brasil

E-mail: oliveiramatheusmoraes@gmail.com

Rubia Carolina Morais Silva

ORCID: https://orcid.org/0000-0002-1191-5172

Universidade Federal do Triângulo Mineiro, Brasil E-mail: rubiacarolina_22@hotmail.com

Davi Leonardo de Souza

ORCID: https://orcid.org/0000-0002-1995-9057

Universidade Federal do Triângulo Mineiro, Brasil

E-mail: davi.souza@uftm.edu.br

\section{Resumo}

Controlar as variáveis de processo no ponto desejado representa desde ganho econômico, qualidade no produto final e controle da segurança industrial. A otimização tem como objetivo a busca pela melhor solução, para que isso ocorra utilizam-se algoritmos como Colônia de Vagalumes e o Evolução Diferencial, os quais minimizam o erro relativo do sinal de saída da variável controlada. Com o objetivo de comparar os algoritmos de evolução diferencial, e o bio-inspirado: colônia de vagalumes e o método clássico de Ziegler-Nichols, foi realizado o projeto de controle PI e de controle PID aplicado em uma planta piloto, considerando dois elementos finais de controle distintos (válvula e bomba) para controle de nível estabelecendo como critério de performance o mínimo esforço possível da variável manipulada. A comparação dos resultados obtidos entre os controladores projetados, utilizando os três métodos para os dois elementos em estudo, demonstrou que os algoritmos de otimização apresentaram um ótimo controle do processo, sem nenhum overshoot (desvio 
máximo do valor da variável controlada, com o valor do setpoint). Os resultados obtidos demostraram que ambos algoritmos de otimização são bons métodos para o projeto de controladores quando trabalhado com o mínimo esforço da variável manipulada.

Palavras-chave: Algoritmo bio-inspirado; Controle feedback; Evolução diferencial; Otimização.

\begin{abstract}
Controlling the process variables at the desired point represents from economic gain, quality in the final product and control of industrial safety. The optimization aims to search for the best solution, so that this occurs, algorithms such as Firefly Colony and Differential Evolution are used, which minimize the relative error of the controlled variable output signal. In order to compare the differential evolution algorithms and the bio-inspired: firefly colony and the classic Ziegler-Nichols method, the PI control and PID control project applied to a pilot plant was carried out, considering two final elements control systems (valve and pump) for level control, establishing the minimum effort of the manipulated variable as a performance criterion. The comparison of the results obtained between the designed controllers, using the three methods for the two elements under study, demonstrated that the optimization algorithms showed an excellent control of the process, without any overshoot (maximum deviation from the value of the controlled variable, with the setpoint value). The results obtained demonstrated that both optimization algorithms are good methods for the design of controllers when worked with the minimum effort of the manipulated variable.
\end{abstract}

Keywords: Bio-inspired algorithm; Feedback control; Differential evolution; Optimization.

\title{
Resumen
}

El control de las variables del proceso en el punto deseado representa desde el beneficio económico, la calidad en el producto final y el control de la seguridad industrial. La optimización tiene como objetivo buscar la mejor solución, para que esto ocurra, se utilizan algoritmos como Firefly Colony y Diferencial Evolution, que minimizan el error relativo de la señal de salida de la variable controlada. Para comparar los algoritmos de evolución diferencial y la bioinspiración: colonia de luciérnagas y el método clásico Ziegler-Nichols, se llevó a cabo el proyecto de control PI y control PID aplicado a una planta piloto, considerando dos elementos finales sistemas de control (válvula y bomba) para control de nivel, estableciendo el mínimo esfuerzo de la variable manipulada como criterio de 
rendimiento. La comparación de los resultados obtenidos entre los controladores proyectados, utilizando los tres métodos para los dos elementos en estudio, demostró que los algoritmos de optimización mostraron un excelente control del proceso, sin ningún sobreimpulso (desviación máxima del valor de la variable controlada, con el valor del punto de ajuste). Los resultados obtenidos demostraron que ambos algoritmos de optimización son buenos métodos para el diseño de controladores cuando se trabaja con el mínimo esfuerzo de la variable manipulada.

Palabras clave: Algoritmo bioinspirado; Control de retroalimentación; Evolución diferencial; Optimización.

\section{Introdução}

Um tanque de armazenamento também conhecido por reservatório é destinado para armazenar fluidos ou ser um recipiente onde ocorrem reações químicas. Para que o rendimento desses tanques seja mais eficaz são empregadas instrumentações que fazem o controle de variáveis desejadas em um processo.

De acordo com Ogata (2010), o controle é essencial para manter os processos em determinados níveis, pressão, temperatura, umidade, entre outras. Para as indústrias manterem essas variáveis no ponto desejado significa desde ganho econômico, melhor qualidade no produto final e controle da segurança industrial.

Esse controle de processo por meio de instrumentação é modelado e definido computacionalmente através de softwares disponíveis como o Scilab ${ }^{\circledR}$ e o SCADA ${ }^{\circledR}$. Um tipo de controle amplamente utilizado é o de feedback ou realimentação, no qual o medidor analisa o valor da variável de saída e compara com o valor desejado, calculando assim o erro que é imediatamente encaminhado para o controlador.

Se o erro for mínimo o setpoint foi atingido, caso não o sistema será repetido até a minimização do erro. Nos controladores feedback os parâmetros são definidos por meio do controlador PID (Proporcional Integral Derivativo) corrigindo os interferentes presentes na saída do sistema. O controle conhecido como PID traz respostas a desvios proporcionais, integrais e derivativos.

Para tornar esse controle mais efetivo deve-se utilizar métodos de otimização e existem inúmeros métodos com essa finalidade, porém, nem todos apresentam a mesma 
aptidão. A otimização é uma técnica que visa obter os valores ótimos de uma determinada função.

Os métodos de resolução de problemas de otimização podem ser classificados em dois tipos, determinísticos (ou clássicos) e não-determinísticos (meta-heurísticos, evolutivos ou randômicos). Com o foco nos métodos não-determinísticos, estes possuem como base para resolver problemas desse tipo a criação de uma população de possíveis candidatos.

A determinação da função de transferência (FT) é fundamental para o estudo da dinâmica e do controle de processo. A FT é definida como relação entre a variável de saída e variável de entrada. A variável de saída ou controlada, é aquela que deseja manter o valor desejado e a variável de entrada (manipulada) é a entrada do processo que é ajustada para manter a variável controlada no valor desejado (setpoint).

Segundo Smith \& Corripio (1997), a representação geral de uma função de transferência é dada pela equação 1:

$$
G(s)=\frac{Y(s)}{X(s)}=\frac{K\left(a_{m} s^{m}+a_{m-1} s^{m-1}+\cdots+a_{1} s^{1}+a_{0}\right) e^{-t_{0} s}}{\left(b_{n} s^{n}+b_{n-1} s^{n-1}+\cdots+b_{1} s^{1}+a_{0}\right)}
$$

Onde, $G(s)$ representa a função de transferência e $Y(s)$ e $X(s)$ as transformada de Laplace da variável de saída e entrada do processo, respectivamente. A constante $\mathrm{K}$ representa o ganho do processo, a e b os coeficientes dos polinômios do numerador e do denominador da função de transferência e $\mathrm{t}_{0} \mathrm{o}$ tempo morto (atraso do sistema).

A equação da função de transferência de um sistema de primeira ordem com tempo morto pode ser mostrada na equação 2 .

$$
G(s)=\frac{K \cdot e^{-\theta s}}{\tau s+1}
$$

em que, $K$ é o ganho do processo, $\theta$ o tempo morto e $\tau$ a constante de tempo. Segundo Seborg, Edgar, Mellichamp e Doyle (2017), para obter tais parâmetros utiliza-se o método gráfico ou método da curva de reação, mostrado na Figura 1. 
Research, Society and Development, v. 9, n. 7, e743974794, 2020

(CC BY 4.0) | ISSN 2525-3409 | DOI: http://dx.doi.org/10.33448/rsd-v9i7.4794

Figura 1: Gráfico para obtenção de parâmetros da função de transferência.

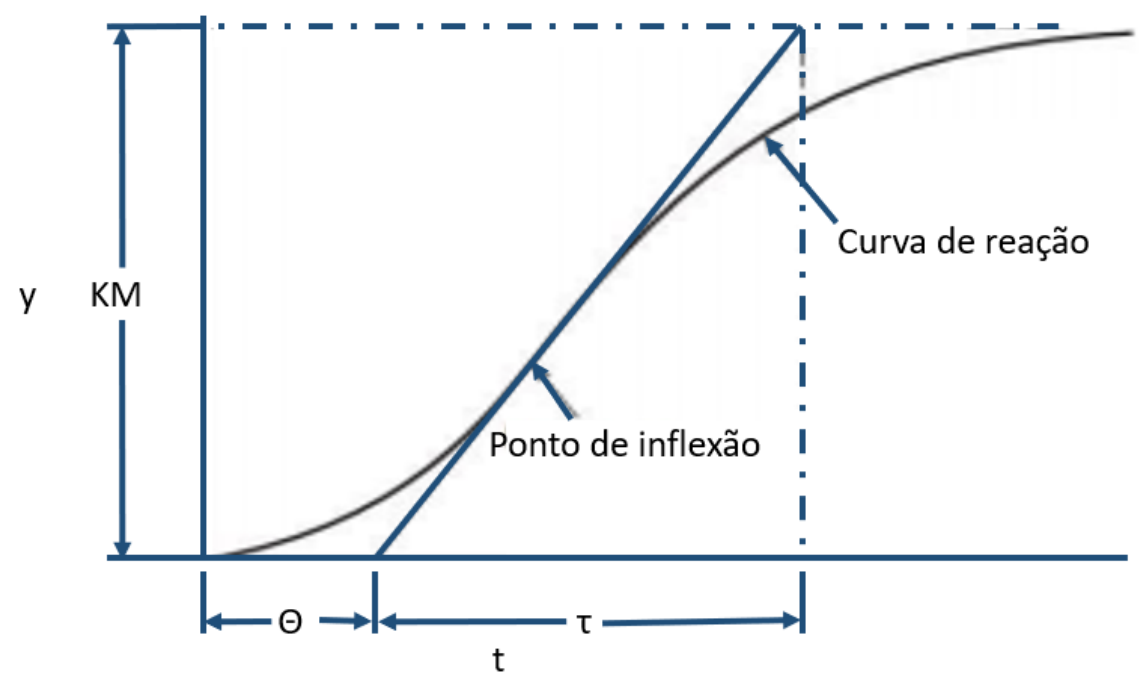

Fonte: Adaptado de Seborg et al.,2017.

Em que, o eixo da abcissa $(t)$ representa a soma do tempo morto $(\theta)$ e da constante de tempo $(\tau)$, enquanto que o eixo da ordenada $(y)$ o produto da constante do ganho $(K)$ com a amplitude do degrau $(M)$.

Um tipo de controle amplamente utilizado é o de feedback, o diagrama de blocos mostrado na Figura 2 representa uma malha fechada de sistema feedback. Cada bloco representa uma função de transferência presente no processo, $G c, G v, G p, G m$ e $G d$ são representações respectivamente da FT do controlador, do elemento final de controle, da planta, do medidor e da perturbação. A variável de saída é representada por Y, a variável de entrada por $Y s p$ e a perturbação do processo é representada por $D$.

Figura 2: Diagrama de blocos para malha fechada de sistema de controle feedback.

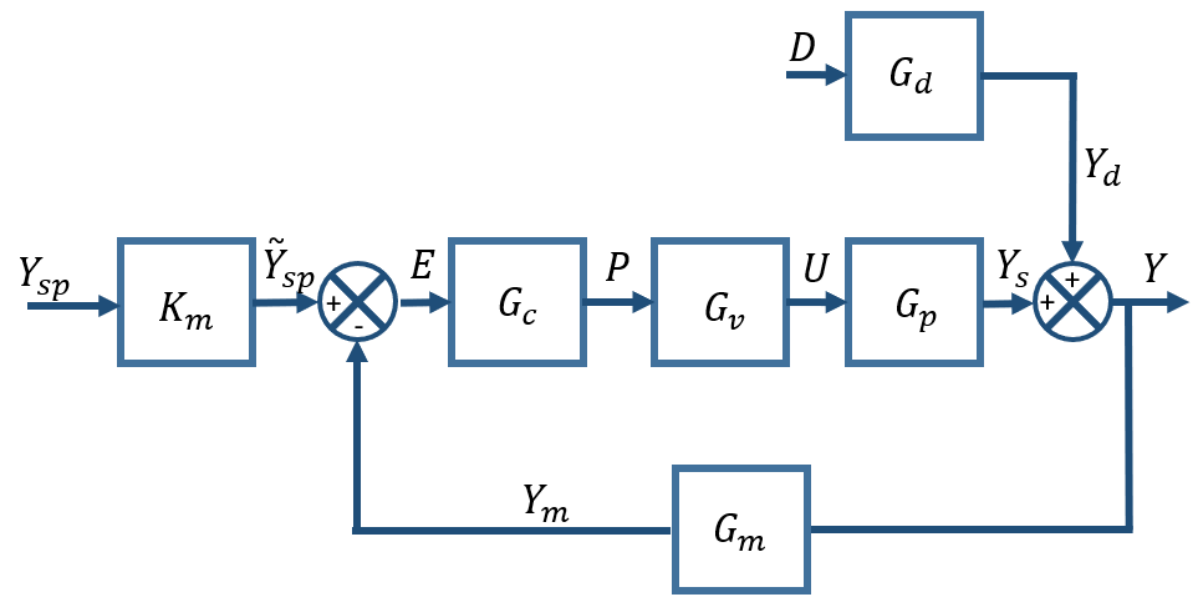

Fonte: Adaptado de Seborg et al.,2017. 
Na Figura 2, o medidor analisa o valor da variável de saída $(Y)$ e compara com o valor desejado ou setpoint (Ysp) calculando assim o erro que é encaminhado para o controlador. Se o erro for mínimo o valor encontrado é o setpoint, mas se o erro não estiver dentro da estimativa acontece novamente o procedimento até que o erro seja minimizado. Este loop contínuo é denominado feedback.

Existem dois sistemas que são provenientes desta malha: servo e regulador. O sistema servo leva em consideração apenas as alterações no setpoint $\left(Y_{s p}\right)$, representado na Equação 3. Enquanto o regulador leva em consideração as perturbações $(D)$, descrito pela Equação 4.

$\frac{Y(s)}{Y_{s p}(s)}=\frac{K_{m} G_{c} G_{v} G_{p}}{1+G_{c} G_{v} G_{p} G_{m}}$

$\frac{Y(s)}{D(s)}=\frac{G_{d}}{1+G_{c} G_{v} G_{p} G_{m}}$

O elemento final de controle, representado por $G_{v}$, tem como função atuar diretamente sobre o processo, recebendo sinal do controlador para então corrigir o desvio do valor desejado. Podem ser válvula, bomba, solenoide e entre outros.

O controlador, representado por $G_{c}$ na malha, pode ser determinado utilizando três ações: proporcional, integral e derivativo (PID). O controle integral corrige os erros ou reduz offset gerados pelo controle proporcional. Se o processo for de alta complexidade, o controle derivativo tem como objetivo corrigir as oscilações contínuas gerados pelo controle integral. Mas em processos de baixa complexidade, a inclusão do derivativo acaba atrapalhando o desenvolvimento do mesmo provocando oscilações.

A representação das três ações do controlador pode ser descrita pela Equação 5.

$$
p=\bar{p}+K_{e}\left[e(t)+\frac{1}{\tau_{l}} \int_{0}^{t} e(t) d t+\tau_{D} \frac{d e}{d t}\right]
$$

Na qual $e(t)$ representa o erro, $p$ a parte proporcional, $K_{e}$ o ganho da função e $\tau_{I}$ e $\tau_{D}$ as constantes de tempo do controle integral e derivativo, respectivamente.

Aplicando a transformada de Laplace na equação 5 obtém-se a função de transferência do controlador PID, descrita pela equação 6. 
$G_{C}=K_{C}\left(1+\frac{1}{\tau_{I} s}+\tau_{D} s\right)$

O controlador descrito na equação 6 é chamado de PID, por apresentar as três ações: proporcional, integral e derivativo. Dependendo do processo em estudo, pode-se utilizar controlador PI (Proporcional Integral) ou apenas P (Proporcional).

No método da curva de reação, Ziegler e Nichols (CRZN) determina modelos que relacionam os parâmetros encontrados experimentalmente pelo método gráfico $(K, \theta$ e $\tau)$ com os parâmetros do controlador PID $\left(K_{\varepsilon}, \tau_{I}\right.$ e $\left.\tau_{D}\right)$. Os parâmetros determinados pelo modelo de CRZN tanto para controle PID quanto para PI são descritos na Tabela 1.

Tabela 1: Parâmetros dos controladores PID e PI do modelo de Ziegler e Nichols.

Expressões para controle PID

\section{Expressões para controle PI}

\begin{tabular}{ccc}
\hline$K_{C}$ & $\frac{1,2}{K}\left(\frac{\theta}{\tau}\right)^{-1}$ & $\frac{0,9}{K}\left(\frac{\theta}{\tau}\right)^{-1}$ \\
\hline$\tau_{I}$ & $2 \theta$ & $3,33 \theta$ \\
\hline$\tau_{D}$ & $0,5 \theta$ & 0
\end{tabular}

Fonte: Adaptado de Seborg et al., 2017.

Para tornar o controle de um processo mais efetivo costuma utilizar métodos de otimização, que corresponde a um mecanismo para trabalhar com a melhor solução, na qual o procedimento compara os resultados com a melhor solução. Grande parte dos problemas existentes possuem mais de um objetivo, e para resolver estes problemas utiliza-se de simulações computacionais e otimizações multiobjetivo.

Os métodos de resolução de problemas de otimização como esses podem ser classificados em dois tipos, determinísticos (ou clássicos) e não-determinísticos (metaheurísticos, evolutivos ou randômicos). Os métodos clássicos possuem uma convergência prematura devido a dificuldade que possuem de lidar com mínimos locais. Enquanto os métodos não determinísticos são métodos evolutivos, com estratégias de solução do problema baseada em comportamentos observados na natureza, ou bio-inspirados. No qual possuem como característica principal encontrar várias soluções ótimas em uma única simulação, devido a sua abordagem de pesquisa baseada na população. 
Um exemplo é o algoritmo colônia de vagalumes (ACV) que é baseado na bioluminescência dos vagalumes. A bioluminescência tem uma influência sobre os vagalumes, já que um vagalume é atraído pelo outro devido a luminosidade que emitem.

Segundo Yang (2010), existem três funções associadas a bioluminescência: i) ferramenta de comunicação e atração para acasalamento; ii) isca para atração de possíveis presas; iii) mecanismo de alerta para potenciais predadores.

O algoritmo proposto por Yang (2010) considera as seguintes hipóteses:

a) Os vagalumes são unissex e podem ser atraídos pelo outro;

b) A atratividade é diretamente proporcional a luminosidade e diminui com o aumento da distância entre eles. Se não houver um vagalume mais brilhante que os demais, todos se moverão aleatoriamente;

c) A luminosidade de um vagalume é estabelecida pela função objetivo.

d)

A intensidade da luz diminui com o aumento da distância entres os vagalumes determinada pela equação 7 .

$I=I_{0} e^{-\gamma r^{2}}$

Na qual $I_{0}$ representa a intensidade inicial, $r$ a distância euclidiana entre os vagalumes e $\gamma$ a absorção da luz. A atratividade é proporcional a intensidade da luz e representa o quanto os vagalumes estão próximos, podendo ser expressa pela equação 8 , onde $\beta_{0}$ é a atratividade inicial em $r=0$.

$\beta(r)=\beta_{0} e^{-\gamma r^{2}}$

A movimentação do vagalume representa o movimento das soluções no espaço de busca do problema. Este movimento depende de três fatores: atratividade ( $\beta$ ), absorção da luz pelo meio $(\gamma)$ e aleatoriedade $(\alpha)$. O movimento do vagalume $i$, em direção ao vagalume $j$ mais brilhante é definida pela equação 9.

$x_{i+1}=x_{i}+\beta_{0} e^{-\gamma r_{i j}^{2}}\left(x_{j}-x_{i}\right)+\alpha$ scale $($ rand -0.5$)$ 
Na qual o primeiro termo representa a posição atual do vagalume, o segundo termo a atratividade do vagalume e a intensidade da luz vista pelos outros vagalumes e por fim o terceiro termo indica o movimento aleatório. Onde $\alpha$ é o fator de aleatoriedade, rand é um gerador de números aleatórios dentro de um intervalo [0;1] e scale é um vetor que garante que o vagalume esteja dentro do domínio especificado.

Escrito em forma de pseudocódigo, o ACV é demonstrado pela Tabela 2.

Tabela 2: Algoritmo Colônia de Vagalumes.

\section{Início}

Definir a função objetivo $\mathrm{J}(\mathrm{x}), \mathrm{x}=(\mathrm{x} 1, \mathrm{x} 2, \ldots, \mathrm{xD}) \mathrm{T}$

Definir os parâmetros do ACV

Para $i=1$ até o número máximo de gerações faça

Calcular a intensidade da luz Ii para xi proporcionalmente a $\mathrm{J}(\mathrm{xi})$

Para $\mathrm{j}=1$ até $\mathrm{o}$ número de vagalumes

Calcular o fator de atratividade

Mover o vagalume j em direção aos vagalumes mais brilhantes

Verificar se o vagalume está dentro dos limites

\section{Fim-Para}

Fim-Para

Pós-processamento e visualização dos resultados

Fim

Fonte: Correa, 2019.

Em que $J(x)$ corresponde a função objetivo, $\left(x_{1}, x_{2}, \ldots, x_{D}\right)$ representam as variáveis de interesse, que o algoritmo fornece ao finalizar o número de iterações. Para a simulação existe a dependência de alguns parâmetros tais como tamanho da população, número de interações, parâmetro de aleatoriedade, parâmetro de atratividade e absorção da luz.

Outro exemplo é o algoritmo evolução diferencial é baseado na evolução natural, tendo como o objetivo manter a diversidade da população e obter a melhor solução. $\mathrm{Na}$ evolução diferencial existem três processos: mutação, cruzamento e seleção.

A população inicial é constituída por Np indivíduos que cobre todo o espaço de busca. Na mutação ocorre a geração de novos indivíduos chamados vetores modificados, pela adição da diferença ponderada entre dois indivíduos aleatórios da população a um terceiro indivíduo. O processo de mutação pode ser descrito na equação 10.

$$
v(i)^{(g+1)}=x_{r 1}^{(g)}+F \cdot\left(x_{r 3}^{(g)}-x_{r 2}^{(g)}\right)
$$


Em que $v(i)$ representa 0 vetor modificador; $x_{r 1}, x_{r 2}$ e $x_{r 3}$ vetores distintos escolhidos aleatoriamente e $F$ fator de perturbação.

O processo de cruzamento tem como objetivo aumentar a diversidade dos indivíduos que sofreram a mutação. Então a criação do vetor experimental se dá pela mistura dos componentes do indivíduo modificador com as componentes de um indivíduo escolhido aleatoriamente, chamado de vetor alvo. A estratégia da criação do vetor experimental está descrita na equação 11 .

$u(i)^{g+1}=\left\{\begin{array}{l}v(i)^{g+1}, \text { se } \operatorname{rand}_{i} \leq C R \\ x_{s}(i)^{g}, \text { se } \operatorname{rand}_{i}>C R\end{array}\right.$

Onde $u(i)$ representa o vetor experimental, $v(i)$ vetor modificador, $x_{s}(i)$ vetor alvo, rand um número gerado aleatoriamente no intervalo [0,1] e $C R$ a probabilidade do cruzamento. A Figura 3 é uma representação do processo de cruzamento de acordo com Boccato, Attux \& Zuben (2009).

Figura 3: Processo de cruzamento.

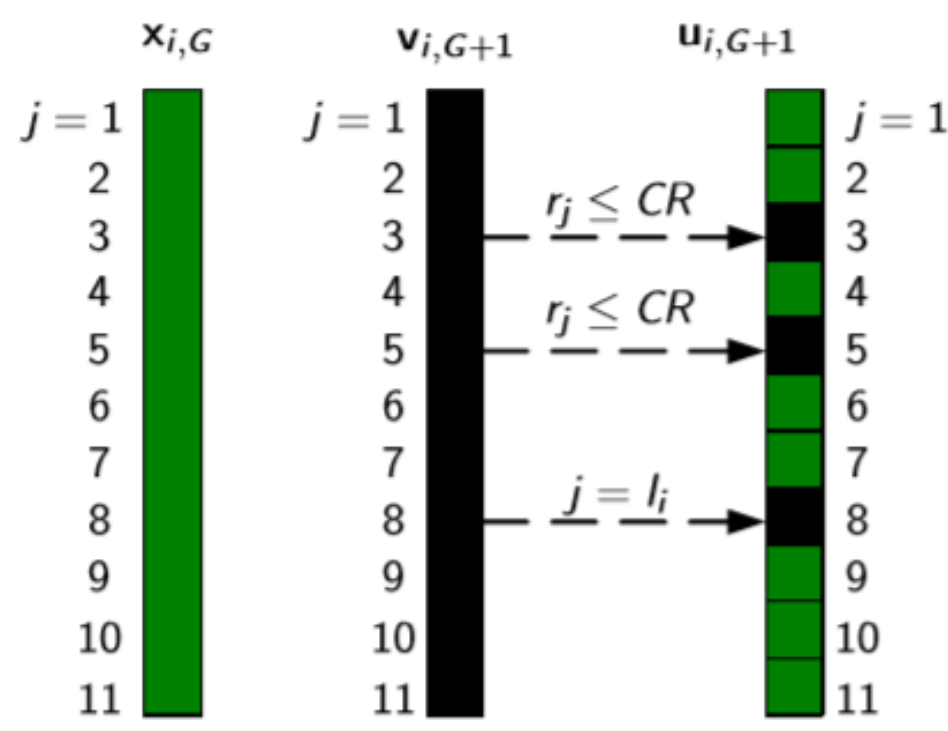

Fonte: Boccato et al., 2009.

Após a etapa de cruzamento, acontece a seleção que tem como objetivo escolher o melhor indivíduo utilizando a função objetivo. Esse processo baseia no estudo da função objetivo, ou seja, se o vetor experimental tiver um menor fitness que do vetor alvo, então na 
próxima geração o vetor experimental será o vetor alvo. Assim o processo de seleção pode ser expresso pela equação 12 .

$$
\left\{\begin{array}{c}
\text { Se } f\left(u(i)^{g+1}\right) \leq f\left(x_{s}(i)^{g}\right) \text {, então } x_{g}(i)^{g+1}=u(i)^{g+1} \\
\text { Se } f\left(u(i)^{g+1}\right)>f\left(x_{g}(i)^{g}\right) \text {, então } x_{g}(i)^{g+1}=x_{s}(i)^{g}
\end{array}\right.
$$

Assim como o ACV, no desenvolvimento do AED (algoritmo de evolução diferencial) há a dependência depende de alguns parâmetros essenciais tais como tamanho da população, fator de perturbação e probabilidade de cruzamento e número de interações.

Conforme descrito no pseudocódigo por Silva, Neto, e Martins (2011), o algoritmo evolução diferencial é demonstrado pela Tabela 3.

Tabela 3: Algoritmo Evolução Diferencial Multiobjetivo.

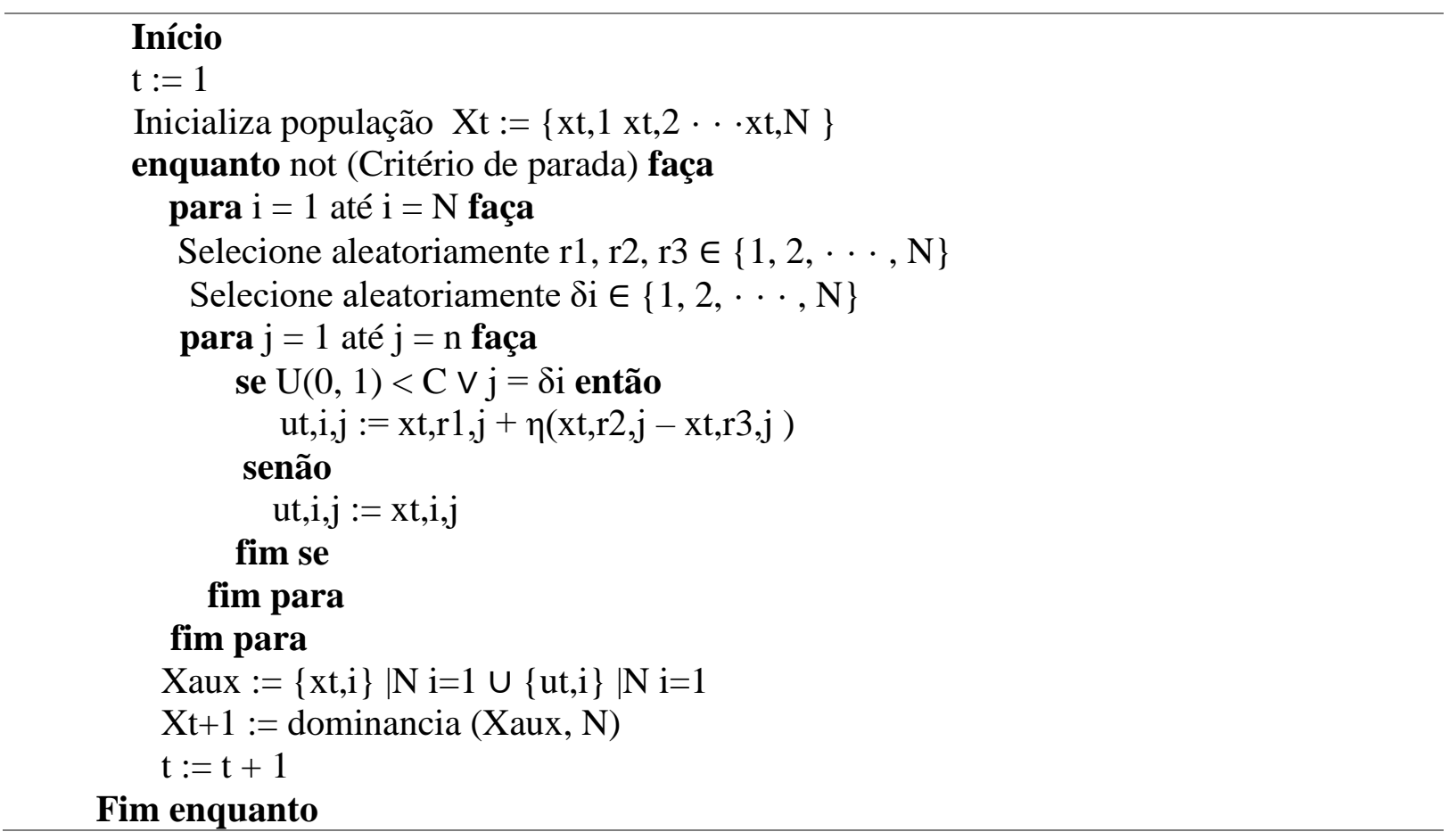

Fonte: Silva et al., 2011.

Portanto o objetivo geral do presente trabalho foi comparar os dois algoritmos metaheurísticos, evolução diferencial e o bio-inspirado (colônia de vagalumes) no projeto de controle PI e PID aplicado em planta piloto, considerando dois elementos finais de controle distintos (válvula e bomba) para controle de nível. 


\section{Metodologia}

\subsection{Planta modelo XL33, fabricante Labtrix ${ }^{\circledR}$}

A planta utilizada para testes está localizada na Universidade Federal do Triângulo Mineiro, no Instituto de Ciências Tecnológicas e Exatas, é demonstrada na Figura 4.

Figura 4: Planta modelo XL33 (a), válvula (b) e bomba (c).

a)

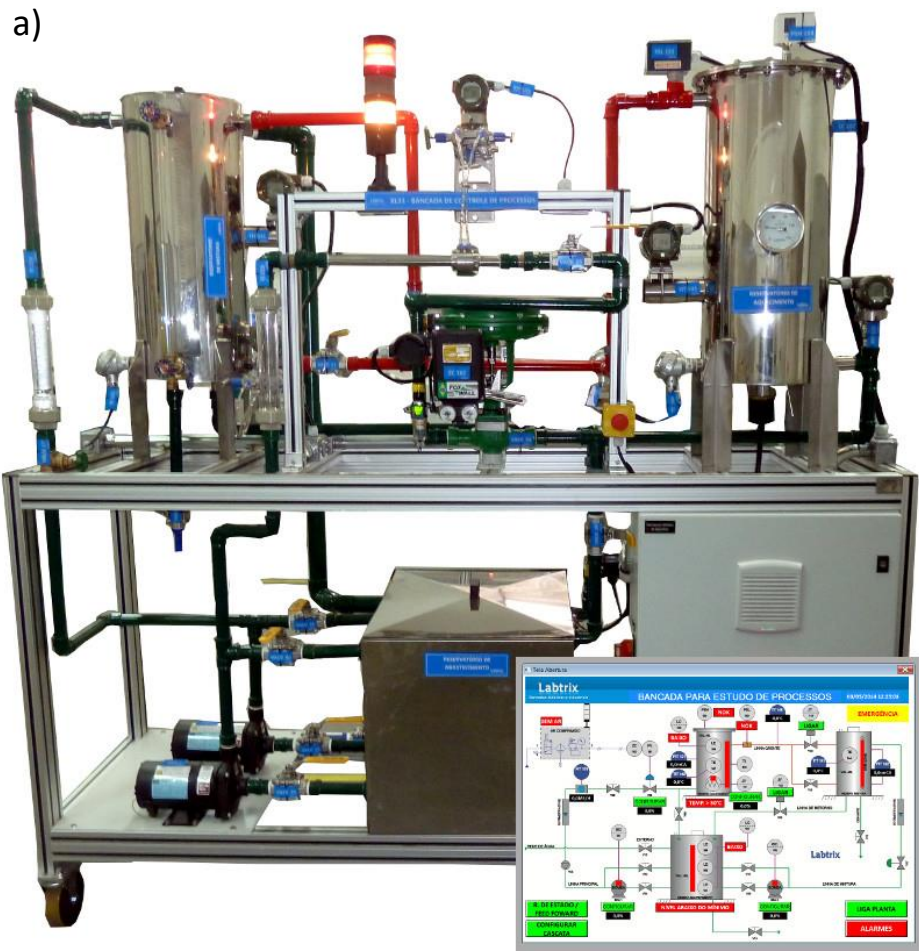

b)

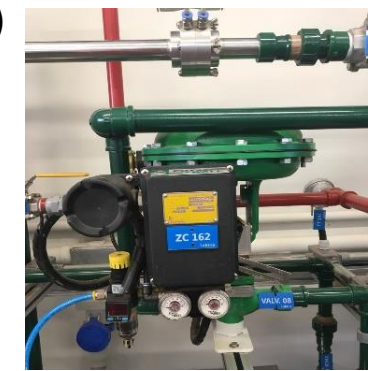

c)

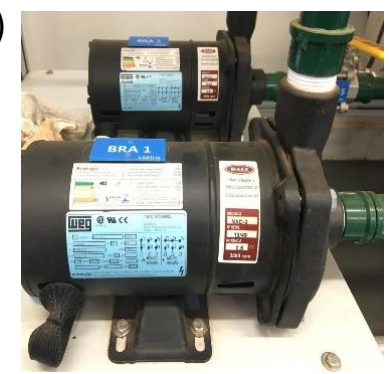

Fonte: Adaptado de Labtrix, 2019.

Para os testes deste trabalho foram utilizados o reservatório inferior e o de nível. O objetivo do estudo é o controle de nível, então foi utilizado a válvula V08 (Figura 4a) e a bomba BRA1 (Figura 4b) como elementos finais de controle. Para o supervisório utilizou-se do software Elipse Scada ${ }^{\circledR}$, adquirido juntamente com a planta.

\subsection{Modelagem da planta}

Para a determinação dos parâmetros do modelo da planta foi utilizado a modelagem clássica através do método gráfico ou método da curva de reação. Este procedimento foi realizado considerando como elemento final de controle a válvula e a bomba. 
Research, Society and Development, v. 9, n. 7, e743974794, 2020

(CC BY 4.0) | ISSN 2525-3409 | DOI: http://dx.doi.org/10.33448/rsd-v9i7.4794

Para a modelagem da planta levando em conta o elemento final de controle a válvula, definiu-se a potência da bomba em $80 \%$, iniciando assim o processo com $25 \%$ de abertura da válvula e com uma vazão de $0,9 \mathrm{~m} 3 / \mathrm{h}$ em um nível de $11,6 \mathrm{~cm}$. Aplicou a perturbação no sistema, abrindo a válvula em $35 \%$ e consequentemente a vazão aumentou-se para $1,15 \mathrm{~m}^{3} / \mathrm{h}$, levando o nível a estabilizar-se em $41,2 \mathrm{~cm}$.

Para a bomba como elemento final de controle, foi utilizado o mesmo procedimento. Primeiramente definiu a abertura a válvula em $40 \%$ e assim iniciou-se a potência da bomba em $64 \%$ e vazão em $0,95 \mathrm{~m}^{3} / \mathrm{h}$, assim o nível inicial foi de $11,4 \mathrm{~cm}$. Aplicando uma perturbação, aumentou-se a potência da bomba para $74 \%$, assim a vazão foi para $1,125 \mathrm{~m}^{3} / \mathrm{h}$ e o nível estabilizou-se em $37,5 \mathrm{~cm}$.

Os dados descritos para a modelagem da planta tanto para bomba quanto para válvula podem ser demonstrados na Tabela 4 .

Tabela 4: Dados de operação e de perturbação da bomba e válvula.

\begin{tabular}{c|c|c}
\hline \multicolumn{3}{c}{ Dados de operação } \\
\hline Grandeza & Bomba & Válvula \\
\hline Potência da Bomba (\%) & 64 & 80 \\
\hline Abertura da válvula (\%) & 40 & 25 \\
\hline Vazão (m³/h) & 0,95 & 0,9 \\
\hline Nível (cm) & 11,4 & 11,6 \\
\hline \multicolumn{2}{c|}{ Dados da perturbação } \\
\hline Grandeza & Bomba & Válvula \\
\hline Abertura da válvula (\%) & 74 & 80 \\
\hline Vazão (m³ dh) & 1,12 & 25 \\
\hline Nível (cm) & 37,5 & 11,6 \\
\hline
\end{tabular}

Fonte: Autores, 2019.

Os pontos experimentais foram coletados a cada 30 segundos, pois o supervisório não possui sistema de aquisição de dados automático. O tempo de amostragem foi 
estabelecido em virtude de testes prévios, notando que a cada 30 segundos não haveria perda de características no comportamento do sistema.

\subsection{Modelo curva de reação Ziegler-Nichols}

As simulações do modelo de curva de reação Ziegler - Nichols (CRZN) foram executadas no software Scilab ${ }^{\circledR}$ utilizando as equações dos parâmetros já estabelecidos pelo modelo. As simulações foram realizadas para os elementos finais de controle, bomba e válvula, e assim projetou-se os controladores PI e PID.

\subsection{Algoritmo de colônia de vagalumes}

Para implementação do algoritmo de colônia de vagalumes utilizou-se o software $\mathrm{Scilab}^{\circledR}$. Definiu-se os parâmetros de acordo com os estudos feitos na área, assim considerouse o tamanho da população $N P=20$; número de interações $\operatorname{Max}_{G \in n}=250$; parâmetro de aleatoriedade $\alpha=0,5$; parâmetro de atratividade $\beta=0,2$ e absorção de luz pelo meio $\gamma=1$

As simulações foram realizadas considerando o elemento final de controle a válvula e a bomba e para cada foram simulados controladores PI e PID. O Algoritmo de colônia de vagalume (ACV) tem como função objetivo minimizar a soma do quadrado do erro do sinal de saída da variável controlada, estabelecida pela equação 13.

$\min =\sum\left(y_{s p}-y\right)^{2}$

\subsection{Algoritmo de evolução diferencial}

Analogamente ao ACV, as simulações do algoritmo de evolução diferencial (AED) foram executadas no Scilab ${ }^{\circledR}$ tanto para a válvula quanto para a bomba, projetando assim controladores PI e PID. Para a execução das simulações do AED, foram definidos os valores dos parâmetros: tamanho da população $N P=20$; número de interações itermax $=250$; fator de perturbação $F=0,8$ e probabilidade de cruzamento $C R=0,8$. 
$\mathrm{O}$ AED tem o mesmo objetivo que o ACV, tem como função objetivo minimizar a soma do quadrado do erro do sinal de saída da variável controlada, estabelecida pela equação 13.

\section{Resultados e Discussão}

Primeiramente, foi obtido a curva de reação para a válvula como elemento final de controle, representada na Figura 5.

Figura 5: Curva de reação para a válvula.

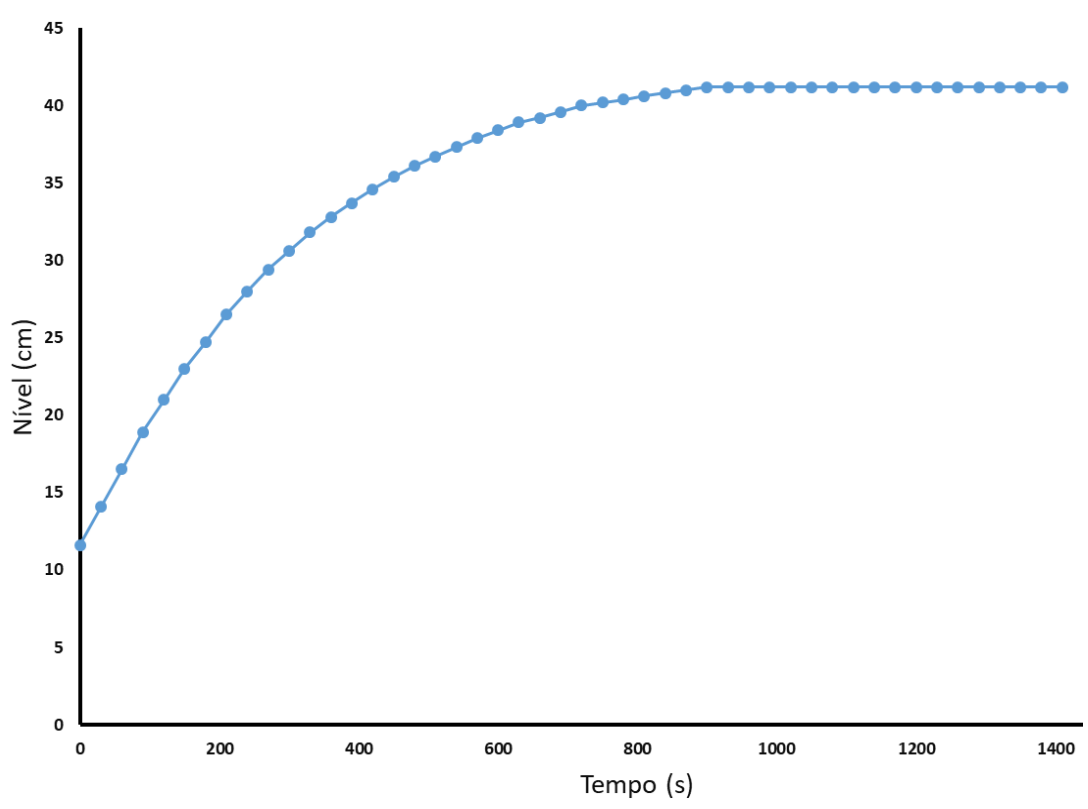

Fonte: Autores, 2019.

Utilizando os conceitos descritos na Figura 1 e relacionando com a Figura 5, foi possível obter os parâmetros da função de transferência da planta de primeira ordem, descritos nas equações 14 e 15.

$$
\begin{aligned}
& K=118,4 \\
& \tau=380 s^{-1}
\end{aligned}
$$

De acordo com o método gráfico, o valor de $\theta$ na curva de reação é zero. Porem para executar o modelo de Ziegler - Nichols é necessário um valor para tempo morto, logo foi utilizado um $\theta$ de 0,8 s para projeto dos controladores. 
Com os parâmetros obtidos, obteve-se a expressão da função de transferência da planta, descrita na equação 16.

$$
G_{p}(s)=\frac{118,4}{380 s+1}
$$

Analogamente a válvula, a curva de reação foi obtida para a bomba utilizando a planta, demonstrada na Figura 6.

Figura 6: Curva de reação para a bomba.

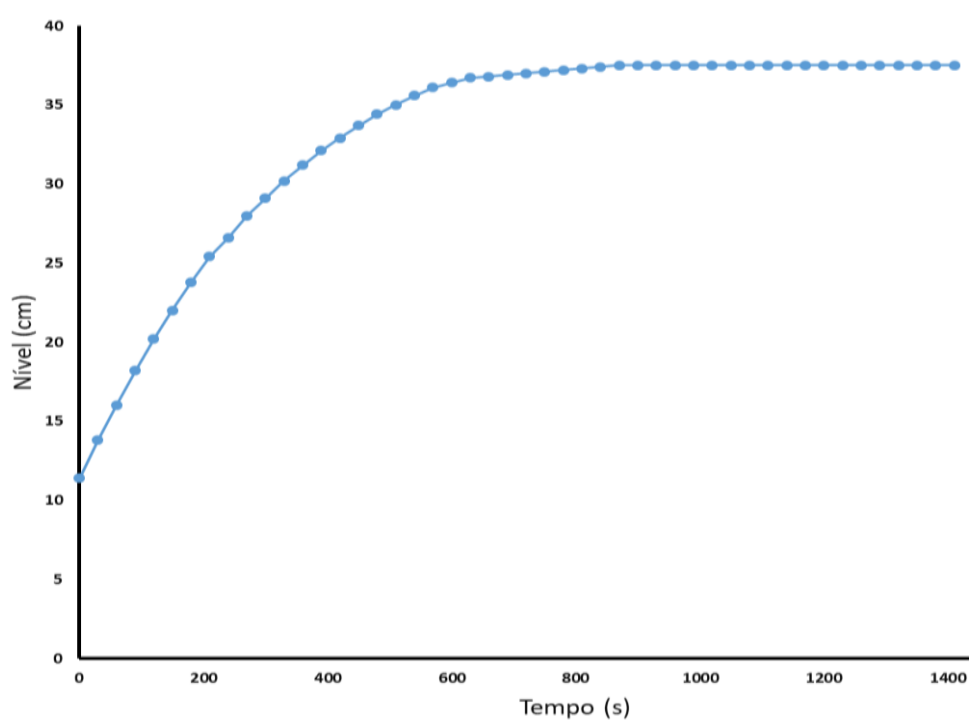

Fonte: Autores, 2019.

Utilizando a curva de reação encontrada para a bomba, foi possível obter os parâmetros da função de transferência da planta de primeira ordem, mostrados nas equações 17 e 18, utilizando o método gráfico descrito na Figura 1.

$$
\begin{aligned}
& K=149,14 \\
& \tau=350 s^{-1}
\end{aligned}
$$

Analogamente a válvula, utiliza-se o mesmo raciocínio para a bomba como elemento final de controle, o valor de $\theta$ na curva de reação é zero. Porem para executar o modelo de Ziegler - Nichols é necessário um valor para tempo morto, logo foi utilizado um $\theta$ de $0,8 \mathrm{~s}$ para projeto dos controladores. 
Research, Society and Development, v. 9, n. 7, e743974794, 2020

(CC BY 4.0) | ISSN 2525-3409 | DOI: http://dx.doi.org/10.33448/rsd-v9i7.4794

Com os parâmetros determinados, obteve-se a equação que representa a função de transferência da planta, descrita na equação 19.

$$
G_{p}(s)=\frac{149,14}{350 s+1}
$$

A partir dos parâmetros da planta foram simulados no Scilab os modelos de CRZN, ACV e AED para a sintonia dos controladores PI e PID, com intuito de determinar os parâmetros dos controladores que estabilizam no valor desejado. Os parâmetros encontrados dos controladores para a válvula como elemento final de controle estão descritos na Tabela 5.

Tabela 5: Parâmetros dos controladores para a válvula.

\begin{tabular}{ccccc}
\hline Modelo & & $\boldsymbol{K}_{\boldsymbol{C}}$ & $\boldsymbol{\tau}_{\boldsymbol{I}}$ & $\boldsymbol{\tau}_{\boldsymbol{D}}$ \\
\hline \multirow{2}{*}{ Ziegler -Nichols } & PI & 3,61 & 2,66 & 0 \\
\cline { 2 - 5 } & PID & 4,81 & 1,60 & 0,40 \\
\hline \multirow{2}{*}{ Colônia de Vagalume } & PI & 20 & 70,28 & 0 \\
\cline { 2 - 4 } & PID & 20 & 64,55 & 0,05 \\
\hline \multirow{2}{*}{ Evolução Diferencial } & PI & 20 & 70,26 & 0 \\
\cline { 2 - 4 } & PID & 20 & 69,70 & 0,05 \\
\hline
\end{tabular}

Fonte: Autores, 2019.

Analogamente a válvula, considerando a bomba como elemento final de controle, obteve-se então os parâmetros dos controladores para os mesmos três métodos, demonstrados na Tabela 6.

Tabela 6: Parâmetros dos controladores para a bomba.

\begin{tabular}{ccccc}
\hline Modelo & & $\boldsymbol{K}_{\boldsymbol{C}}$ & $\boldsymbol{\tau}_{\boldsymbol{I}}$ & $\boldsymbol{\tau}_{\boldsymbol{D}}$ \\
\hline \multirow{2}{*}{ Ziegler -Nichols } & PI & 2,64 & 2,66 & 0 \\
\cline { 2 - 5 } & PID & 3,52 & 1,60 & 0,40 \\
\hline \multirow{2}{*}{ Colônia de Vagalume } & PI & 20 & 69,35 & 0 \\
\cline { 2 - 4 } & PID & 20 & 67,28 & 0,05 \\
\hline \multirow{2}{*}{ Evolução Diferencial } & PI & 20 & 69,35 & 0 \\
\cline { 2 - 5 } & PID & 20 & 67,28 & 0,05 \\
\hline
\end{tabular}

Fonte: Autores, 2019.

Os parâmetros dos controladores encontrados através das simulações foram então implementados na planta, um de cada vez. Foram coletados os dados no tempo de 30s, 
obtendo assim o gráfico representativo utilizando a válvula como elemento final de controle, representado na Figura 7.

Percebe-se pelos valores que o método clássico apresentou valores bem abaixo dos demais métodos, isso se dá devido à dificuldade que esse tipo de técnica tem em trabalhar com mínimos locais, pois apresentam uma convergência prematura. Enquanto os demais apresentam valores maiores depois de ter feito comparações de inúmeras soluções, mostrando a predição e exatidão das técnicas.

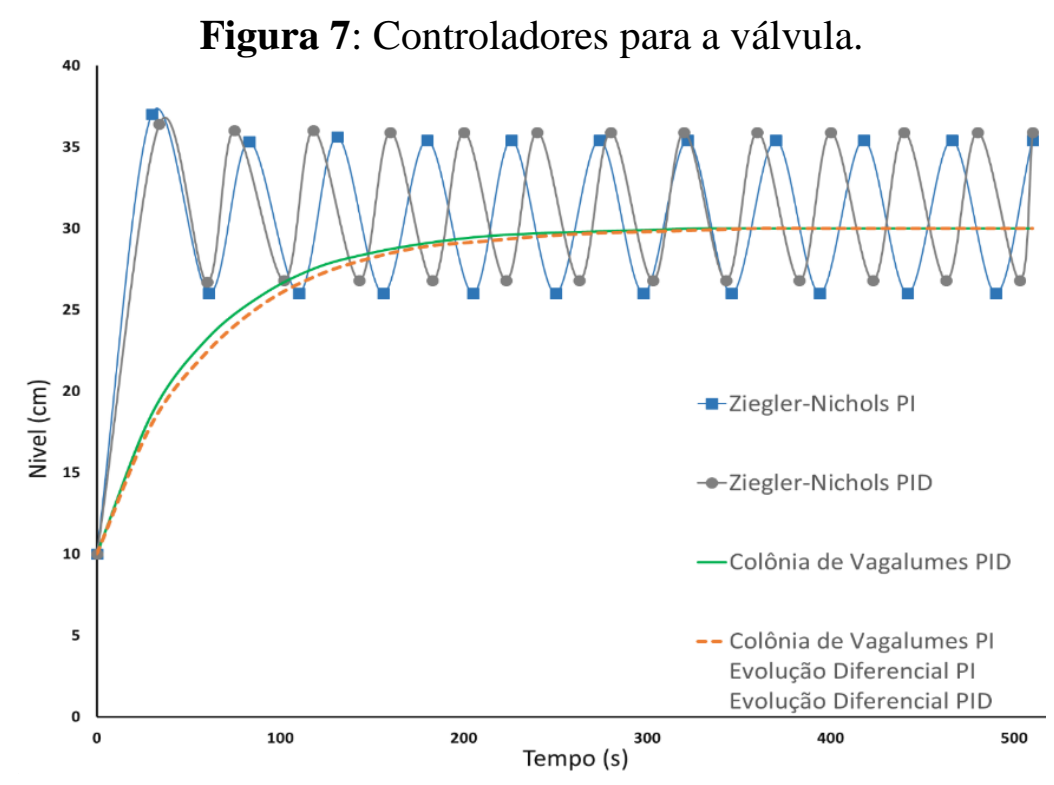

Fonte: Autores, 2019.

Analisando os resultados experimentais obtidos, pode-se perceber que o controlador implementado pelo método de Ziegler-Nichols não apresentou o comportamento esperado, uma vez que o estado estacionário não foi atingido. Pois este método se baseia em um quarto de decaimento, o que faz geralmente a resposta ser oscilatória como ocorreu tanto para o controlador PI como para o PID. Diferente dos outros quatro controladores em estudo que não tiveram nenhum overshoot (desvio máximo do valor da variável controlada, com o valor do setpoint) e controlaram o sistema no setpoint desejado de $30 \mathrm{~cm}$.

O controlador PID otimizado pelo algoritmo de colônia de vagalumes foi o que apresentou o menor tempo de controle de aproximadamente 330s, seguido pelo também PID porém otimizado pelo algoritmo evolução diferencial com um tempo de controle de $360 \mathrm{~s}$, e por fim, o PI de colônia de vagalumes com 360s e evolução diferencial com 390s.

O controlador PID por colônia de vagalumes apresentou uma exigência maior do sistema, podendo analisar que seu comportamento foi acima dos demais enquanto os 
controladores PI e PID por evolução diferencial se apresentaram mais conservadores e mesmo assim alcançaram o setpoint rapidamente quando submetido à perturbação.

Analogamente a válvula, o mesmo processo foi executado para a bomba obtendo-se assim o gráfico das respostas do sistema para a atuação de cada controlador projetado controladores, demonstrado na Figura 8.

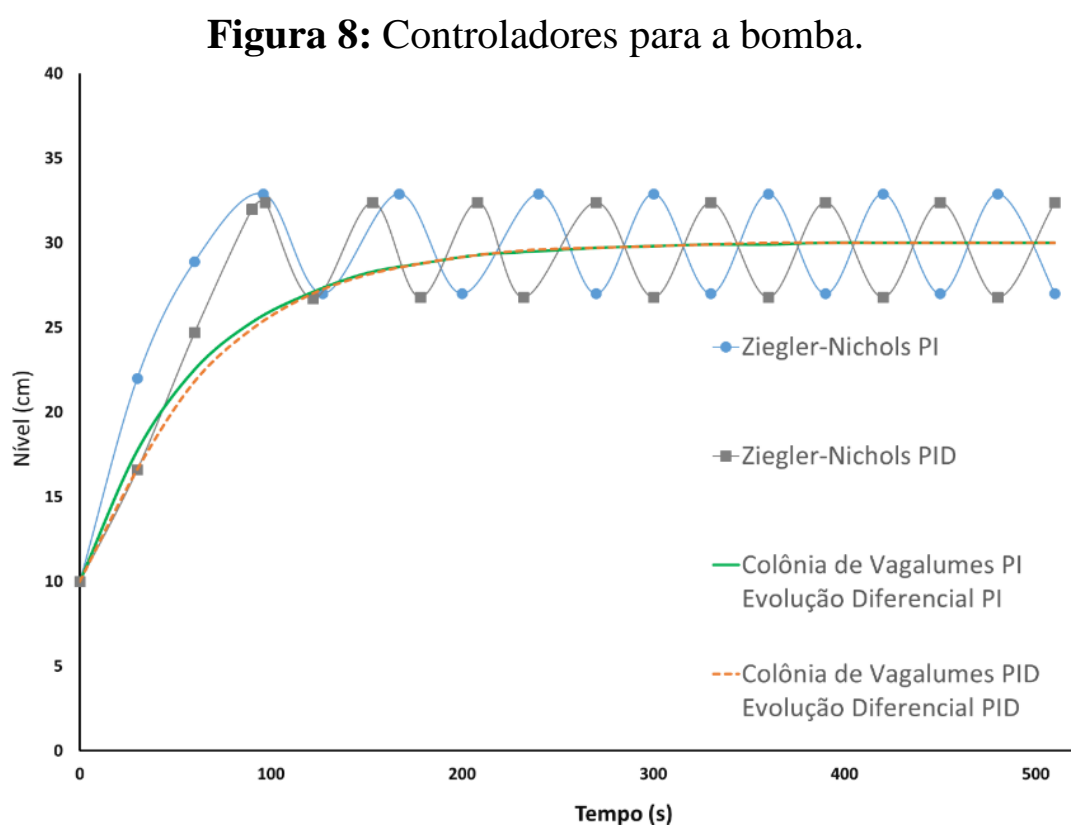

Fonte: Autores, 2019.

Fazendo a análise da mesma forma que foi realizado para a Figura 7. Os dados obtidos, demostra que novamente os controladores projetados pelo método de Ziegler-Nichols não controlaram o sistema no setpoint de $30 \mathrm{~cm}$, também apresentando ação oscilatória tanto para o PI quanto para o PID. Da mesma forma, os outros quatro controladores estudados apresentaram controle do sistema sem apresentar nenhum overshoot, o que é bom, visto que não realiza muito esforço do sistema.

Com relação ao tempo de controle os dois controladores PI apresentaram aproximadamente 390s necessários para controlar em $30 \mathrm{~cm}$ de nível, enquanto os controladores PID gastaram 360s para atingir o setpoint e sendo mais conservadores que os PI, realizando o mínimo esforço da variável manipulada.

Para sistemas não muito complexos e que não apresentam offset é normalmente utilizado controlador PI pois geralmente não possui a necessidade corrigir offset. Este sistema em estudo não é um caso complexo, então a utilização de um derivativo poderia causar algumas oscilações, mas devido as condições de processos pode-se perceber que o PID não 
afetou negativamente o sistema, apenas contribuiu para torná-lo conservador como o desejado.

O método de Ziegler-Nichols necessita de tempo morto, para projeto dos controladores foi utilizado um $\theta$ de $0,8 \mathrm{~s}$. E para os métodos de colônia de vagalumes e evolução diferencial ambos foram realizados sem tempo morto, conforme os modelos das equações 16 e 19.

\section{Considerações Finais}

Com o intuito de comparar algoritmos evolução diferencial, colônia de vagalumes e o método clássico de Ziegler Nichols no projeto de controle PI e PID. O método clássico não se mostrou satisfatório a princípio para o controle de nível da Planta Piloto XL33, considerando a não utilização de sintonia fina, enquanto os controladores utilizando otimização foram satisfatórios, apresentando uma excelente base de resultados.

Estabelecendo como critério de performance o mínimo esforço possível da variável manipulada. Resultou-se que as respostas não apresentaram overshoots, sendo algo interessante visto que se buscava por um sistema conservador, além de que os controladores demonstraram um tempo médio de assentamento de 330s, que corresponde a um tempo aceitável para controle de nível. Conclui-se também que ambos algoritmos de otimização são bons métodos para o projeto de controladores.

O supervisório utilizado na planta piloto não possui sistema de aquisição de dados automático, ou seja, ao final da execução do processo, não há a possibilidade de exportar os pontos experimentais, que neste trabalho, foram coletados a cada 30 segundos, escolhido após testes prévios, e sem perda de características do comportamento do sistema, conforme visualizado nas Figuras 5, 67 e 8. Esta é a principal limitação do processo abordado neste trabalho, sendo assim, faz-se necessário para implementação do sistema de aquisição automático, no futuro, possibilitando um maior número de pontos, e melhor observação do comportamento do sistema.

Outra abordagem para trabalhos futuros, seria o estudo da implementação de um monitoramento do sistema de controle, capaz de detectar e localizar falhas. 


\section{Referências}

Boccato, L., Attux, R. R. F., \& Zuben, F. J. V. (2009). Evolução Diferencial: Introdução e Conceitos Básicos. Recuperado de:

http://scholar.googleusercontent.com/scholar?q=cache:95Wqnjr8uHEJ:scholar.google.com/\& $\mathrm{hl}=\mathrm{pt}-\mathrm{BR} \&$ as_sdt $=0,5 \&$ as_vis $=1$

Correa, V. (2019). Projeto de Controlador PID Cascata em planta piloto, utilizando algoritmo de otimização Bio-inspirado. Trabalho de Conclusão de Curso na Engenharia Química -UFTM Uberaba.

Elipse SCADA. (2020). (Versão 5.1) [Programa de computador]. Kaohsiung - Taiwan: Elipse.

Labtrix. (2019). Portfolio Labtrix - Bancada para ensino de bancada. Recuperado de: http://www.labtrix.com.br/portfolio-item/xl33-bancada-para-ensino-de-controle-de-processos.

Ogata, K. (2010); Engenharia de controle moderno (5 ed). Pearson Prentice Hall. Always Learning. 822p.

Scilab - Algos development. (2020). (Versão 6.1.0) [Programa de computador]. Rungis França: Scilab.

Seborg, D. E., Edgar, T. F., Mellichamp, D. A. \& Doyle, F. J. (2017). Process Dynamics and Control (4ํ ed). New Jersey: John Willey and Sons. 515p.

Silva, C. J., Neto, O. M. N. \& Martins, F. V. C. (2011). Utilização de algoritmo de evolução diferencial multiobjetivo no projeto de controladores. Departamento de Engenharia Elétrica UFMG, In: X Congresso Brasileiro de Inteligência Computacional - CBIC, Ceará., 7p.

Smith, C. A \& Corripio A. (1997). Principles and Practice of Automatic Process Control (2o ed). John Willey and Sons. 768p. 
Research, Society and Development, v. 9, n. 7, e743974794, 2020

(CC BY 4.0) | ISSN 2525-3409 | DOI: http://dx.doi.org/10.33448/rsd-v9i7.4794

Yang, X. S. (2010). Engineering Optimization: An Introduction With Metaheuristic

Applications ( $1^{\circ}$ ed). United Kingdom: John Willey and Sons. 347p.

Porcentagem de contribuição de cada autor no manuscrito

Matheus Divino Moraes Oliveira - 33,35\%

Rubia Carolina Morais Silva - 33,35\%

Davi Leonardo de Souza - 33,30\% 